\title{
FROM RUN OF MINE TO STEEL HOLISTIC APPROACH TO PROCESS OPTIMIZATION*
}

\author{
Christoph Aichinger ${ }^{1}$ \\ Bernhard Hieb/2 \\ Reinhard Redl ${ }^{3}$
}

\begin{abstract}
The optimization and improvement of a modern integral iron and steel works gets more and more difficult as each individual plant is already operated at its best efficiency and at the lowest operational expenditures. However very often the individual plants are considered as stand-alone units and there are losses and inconsistencies on interfaces and take over points, which could be avoided and by that improving the overall performance of the iron and steel works as a holistic unit. Primetals Technologies looked into this subject extensively in the recent years and is now ready to offer new tools and services in order to assist operators to find further potential for improvement in processes, which seem to be fully developed at first sight.
\end{abstract}

Keywords: Metallurgical test work.

\footnotetext{
Vice President Agglomeration, Primetals Technologies, Linz, Austria.

Senior Expert Pelletizing, Primetals Technologies, Linz, Austria.

Technical Sales, Primetals Technologies, Linz, Austria.
} 


\section{INTRODUCTION}

The efficiency of the iron and steel production becomes increasingly a very important factor for any producer in the world. Looking at the overall costs for steel production, the source and quality of iron ore is the most important factor, which influences the overall efficiency downstream to the steel making process. By improving the iron ore quality the entire processing chain benefits because the separation of the impurities accounts for a significant portion of the processing costs in the blast furnace or the melt shop, where the impurities are separated in form of slag, which requires thermal respectively electric energy.

In order to accommodate with these requirements Primetals Technologies intensified recently the activities in the field of beneficiation and developed in parallel a single calculation model, which considers the complete chain of processing plants from the incoming raw materials to the liquid steel with the accuracy of a specific mass balance for any of the individual plants.

With the overall evaluation of the iron and steel making process Primetals Technologies is now in the position to offer all available industrial scale proven plant types from run of mine to liquid steel including beneficiation as single units and also to calculate the complete processing route in one model in order to achieve highest efficiency and lowest processing costs of an integrated iron and steel works.

\section{BASIC AND FACTS}

There are various routes to come from an iron ore to liquid steel, which are shown in figure no. 1.

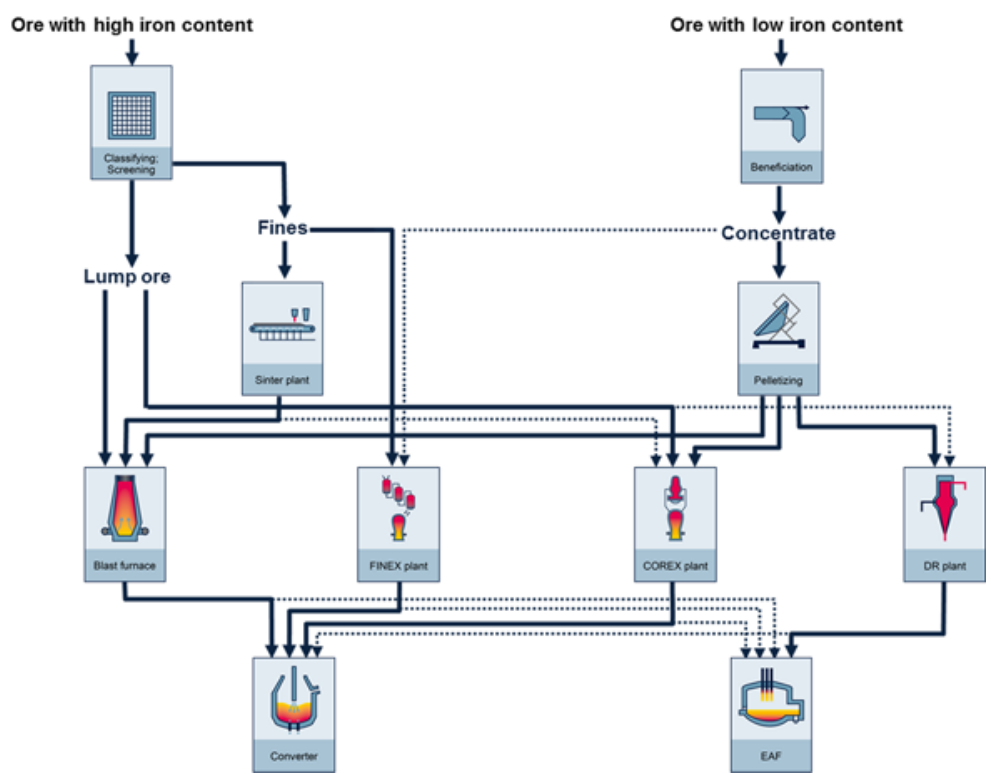

Figure no. 1: Routes for iron and steel making

The processing route, which is implemented or envisaged for a future investment depends very much on the source of iron ore, which is available, but also on other factors like the available type of fuel, the cost of electric power, etc. Considering all these other facts as given for a certain country or location, the quality and properties of the iron ore is a factor, which can be influenced by the type and intensity of beneficiation, which is applied. 
Commonly beneficiation of iron ore is done at the mine site and the iron ore is transported to the customer, who directly feeds it to the downstream plants without any further treatment. This mainly applies to lump ore respectively fine ore, which is used as sinter feed for the conventional blast furnace - BOF steel making route. As the possible upgradation of the iron ore quality at such coarser grain sizes is limited, beneficiation does not have a very important role for this route.

As soon as it comes to concentrate fineness, which enables the upgrading of low quality iron ores to high quality iron ore feed for pelletizing, the importance of beneficiation increases. At grain sizes below $100 \mu \mathrm{m}$ the impurities are separated from the iron ore in different processes and methodologies depending on the mineralogy of the iron ore. Beneficiation plants comprises generally the sequence of several processing technologies like comminution, classification, gravity separation, density separation, magnetic separation, sizing and/or flotation. The final product is determined by the order of the processing technologies.

In the recent years steel making in the EAF was for a huge portion scrap based, however due to the unpredictable price of scrap and in particular of high quality scrap, many customers started investigating on DRI as feed for their EAF. Additionally the DRI route attracted many customers, who already sell iron ore or pellets to the market and now want to extend downstream by producing $\mathrm{HBI}$ as merchant good.

For all such plants and projects the availability of high (DR-)grade pellets is of highest relevance in order to produce high quality DRI for boosting steel making or $\mathrm{HBI}$ as sellable product. While pelletizing plants same as direct reduction plants are processing steps, in which the impurities like Silica and Alumina are just passing through, they could already be separated in the beneficiation plant and by that resulting in an energy saving over the whole process route. The less impurities, which are charged into the EAF, the less slag needs to be separated and therefore the less power is required for steel making. Such scenario is much more important for countries or locations with very high costs for power, which means that more focus should be given in higher efforts for the beneficiation, where the costs for power are negligible in comparison with the EAF process. On the other side a higher degree of beneficiation always means a certain increased loss of iron ore carriers to the tailings, which has to be balanced with the reduced costs for power. This results from the fact, that the minerals like hematite/magnetite, silica or alumina are always intergrown to each other in its natural appearance and the finer a material is ground the better a mineral can be liberated.

\section{HOLISTIC APPROACH TO PROCESS OPTIMIZATION}

\subsection{Technological Test Work}

An extensive practical test work is by that a key factor in order to learn about the mineralogical composition and the separation behaviour of a certain raw material and subsequently for developing the optimum processing route for the raw material. Beneficiation can follow various different routes and methodologies, therefore a wide spectrum of equipment and testing facilities is required to make a comprehensive test work of a raw material. A selection of the apparatuses, which are available in the Primetals laboratory is shown in figure no. 2. 


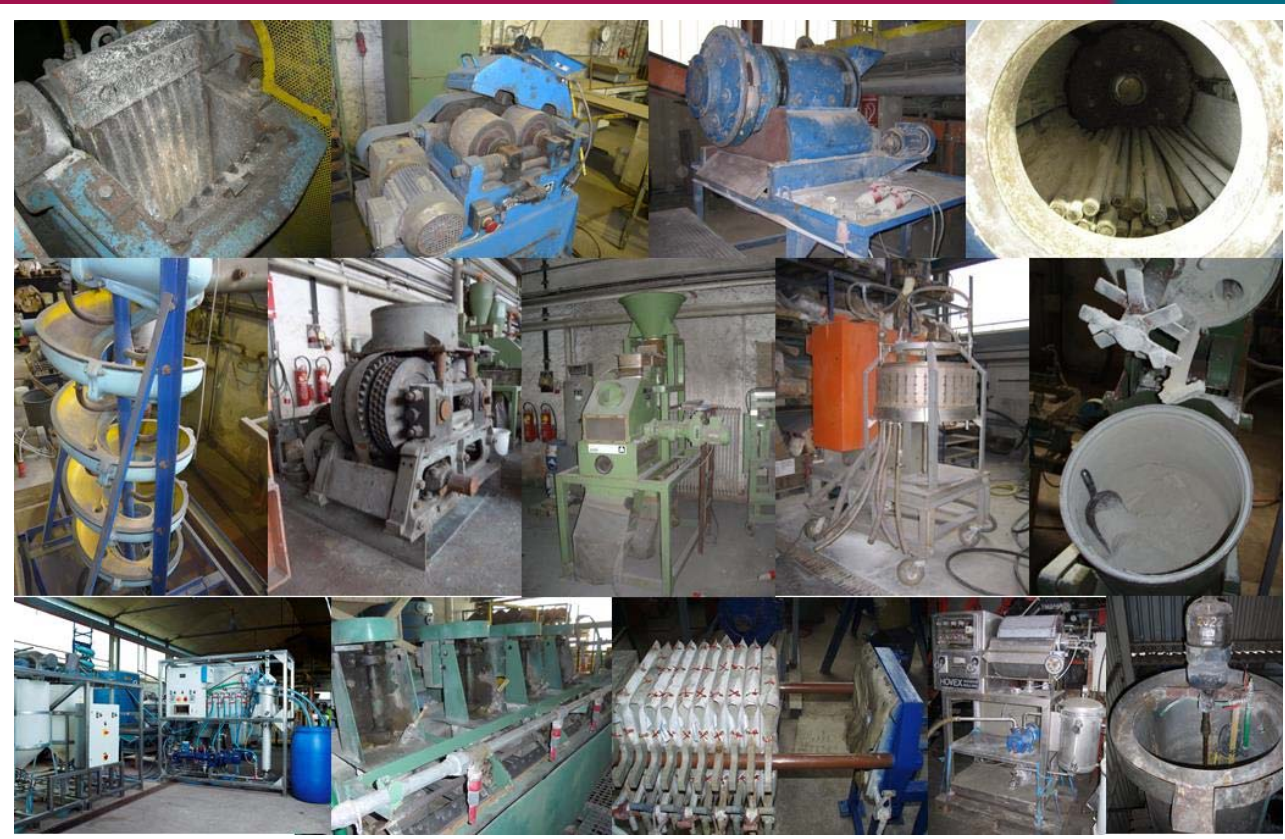

Figure no. 2: Various apparatuses at PT laboratory for extensive beneficiation test work

Downstream to the beneficiation test laboratory a pot grate test facility is available, which has been commissioned successfully in 2014 .

The test facility is fully automated and provides with state of the art automation hardware and software to simulate all typical steps of the travelling grate pelletizing process. For up-draft drying ambient air is heated by an electrical heater while for down-draft drying, pre-heating, firing and after-firing hot gas is generated by a natural gas burner system. The oxygen content of the hot gas is controlled by pure oxygen injection, needed for oxidation reactions occurring in the induration process. For each process step duration, gas temperature, pressure above and below the pellet filling as well as oxygen content of the hot gas are preset on a respective parameter input sheet. The temperature profile for the pre-hating and firing phase is set by 5 base points each. The input parameters can be set freely and allow conventional but also special process configurations for e.g. very high sulfur bearing ores.

The indurating chamber of the test facility is a refractory lined cylinder with a diameter of $240 \mathrm{~mm}$, corresponding to $\sim 15 \mathrm{~kg}$ of hearth layer and $\sim 35 \mathrm{~kg}$ green pellets fired per test. Several thermocouples record the temperatures in the pellet bed at different layers. Pressure indicators above and below the pellet pot regulate the fans, which is the basis for simulating the different zones of the travelling grate process. The temperature profile above the bed is controlled by another thermocouple, which regulates the hot gas system. The off-gas composition is monitored and recorded in an online gas analyser, this and various other trends and graphs of a test can be displayed at any time during and after the test work.

Figure no. 3 shows pictures and a schematic view of the pot grate test facility as well as a typical recording sheet of one pot test. Based on these data the design of a new pelletizing plant or the upgrade of an existing pelletizing plant are developed by using them in a process simulation model, in which the relevant process and plant parameters are calculated and exported. 


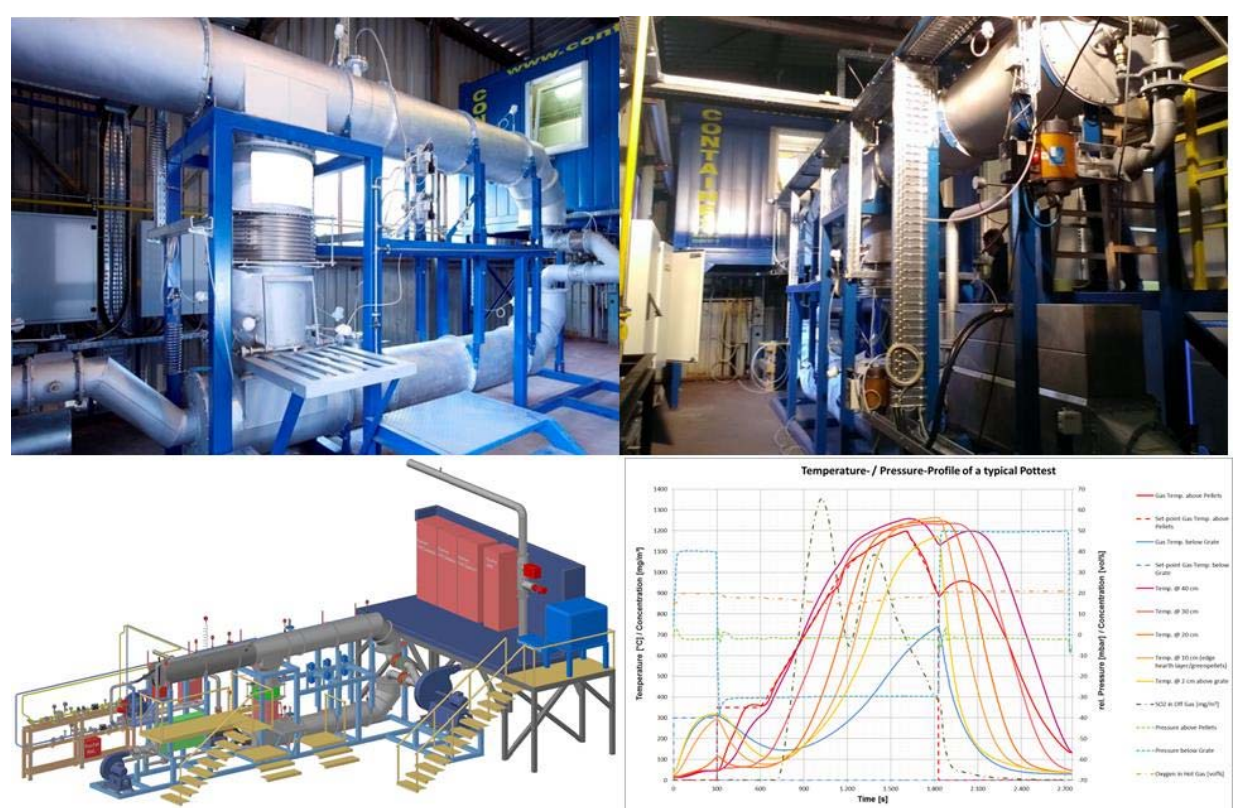

Figure no. 3: Pot grate test facility

The laboratory facilities are completed by several apparatuses for reduction and smelting tests of different types and sizes. This set up allows the reproduction of all important processing routes for iron and steel production in laboratory scale, semipilot scale and in some cases even in pilot scale, so that practically all customer requirements and applications can be fulfilled. In figure 4 some of the available facilities for reduction and smelting tests are shown.

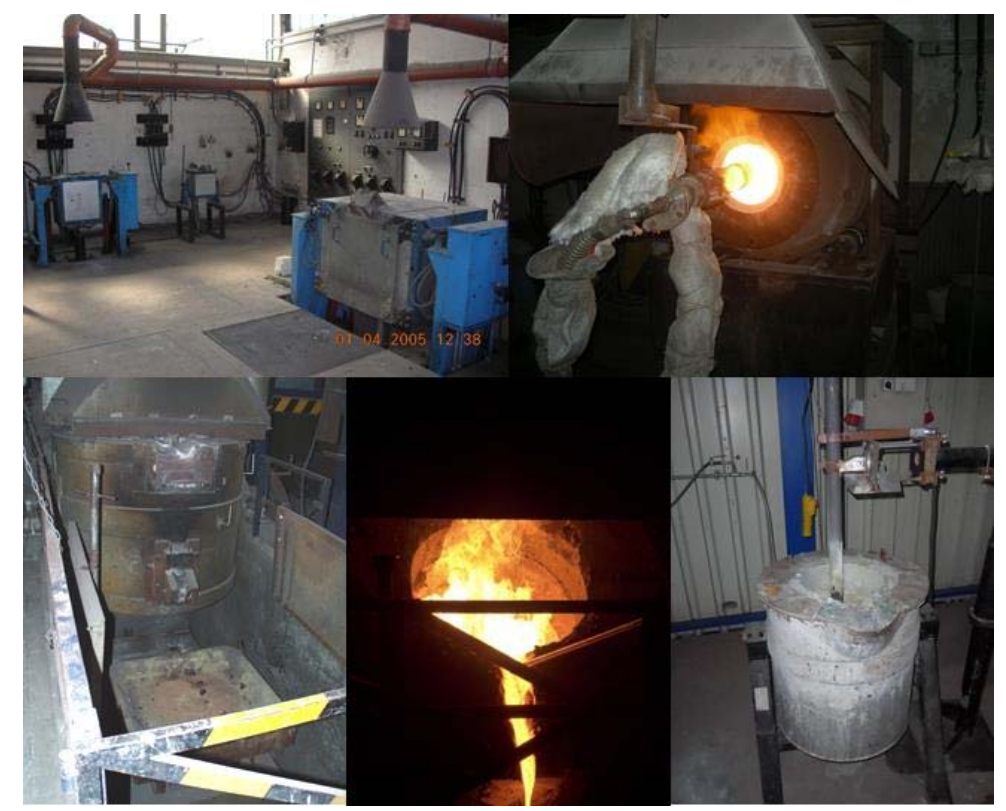

Figure no. 4: Reduction and smelting test facilities: different sizes of apparatuses available

\subsection{Integrated Iron and Steel Works Calculation Model}

In parallel to the practical test work for processing incoming run of mine ore to liquid steel in the laboratory, a theoretical model has been developed, which combines the different process steps in one calculation. Generally it is common, 
that plant operators calculate each plant as stand-alone unit and do not consider the iron and steel making process as a whole, which leads to losses in terms of efficiency and flexibility, which at the end results in higher OPEX. The calculation model offers a holistic approach for all different processing routes with the target to optimize each process by streamlining it with the upstream as well as downstream processes and by that reduce the overall OPEX of an integrated iron and steel works. The basis for this development was the availability of detailed thermodynamic process calculation models for all types of plants in the iron and steel industry (i.e. beneficiation plants, sinter plants, pelletizing plants, blast furnace, BOF shop, etc.). These calculations were combined to a single mathematical model, which now can be made available and adjusted for all different customer requirements within a very short period of time.

Primarily the calculation model is aimed at two applications, which are described in following examples:

Determination of the master plan for green field project development respectively brown field extension projects by comparing different plant routes: such application is e.g. the comparison of a blast furnace / BOF with a Midrex / EAF route for a certain location.

The second application is the optimization of operating plants, which requires a close cooperation between plant operators team and the Primetals specialists for setting up the specific calculation model according to the as-is status of an integrated iron and steel works. After having the model available the consequences of any operational change in the whole works can be predicted for all other plant parts accurately and quickly and countermeasures, if required, initiated immediately. By that long-term, cost-involving adjustment processes in the neighboring plants of the integrated iron and steel works can be shortened and expensive real plant trials are not necessary anymore at all.

With the model in place answers to questions like the effect of change of raw material on slag quality \& quantity in BOF melt shop, the effect of limestone addition in pelletizing plant on power consumption in EAF or the requirements on pellet quality for achieving specific coke consumption in blast furnace can be made directly, which offers a completely new dimension in controlling the operational relations of an integrated iron and steel works.

The calculation model itself has been programmed on a flexible software (gPROMS), which has been adapted for the iron- and steel industry. The software offers several additional features, which supplement to the primary benefits, which are mentioned above.

These are

- Calculation of full mass- and energy balance over the entire production route as well as over each single process step in one and the same model

- Forward and backward calculation possible, this means that it is also possible to calculate from a fixed end product backward to the raw material requirements

- Detailed generation of results and transfer to EXCEL reporting sheet

- Short calculation time (<5 minutes )

- User friendly layout and input/output sheets adapted to steel industry practice

- Direct flowsheet generation

In the metallurgical library of the calculation model around 250 different models have been considered, which do not cover the principle metallurgical processes like a pellet plant or a blast furnace only but also include all possible peripheral processes 
like for heat or waste heat integration, heat exchanger, gas cleaning plants, $\mathrm{CO}_{2}-$ sequestration, etc. This is very important when it comes to questions like the improvement of the environmental footprint of a plant. Another aspect in this regard is the utilization of all steel plant wastes respectively by-products, which are generated in an integrated iron and steel works. Recycling loops can be easily added, changed or deleted and the operational results compared. The correctness of the various models has been validated at numerous running plants so that the correctness and the reliability of the calculations is confirmed.

The following figure shows a typical output flowsheet of the plant calculation model for the classical blast furnace / BOF route. Various recycles are considered in the plant configuration. There is no limitation to the complexity and the number of different individual plant units, which can be considered respectively included in one calculation model.

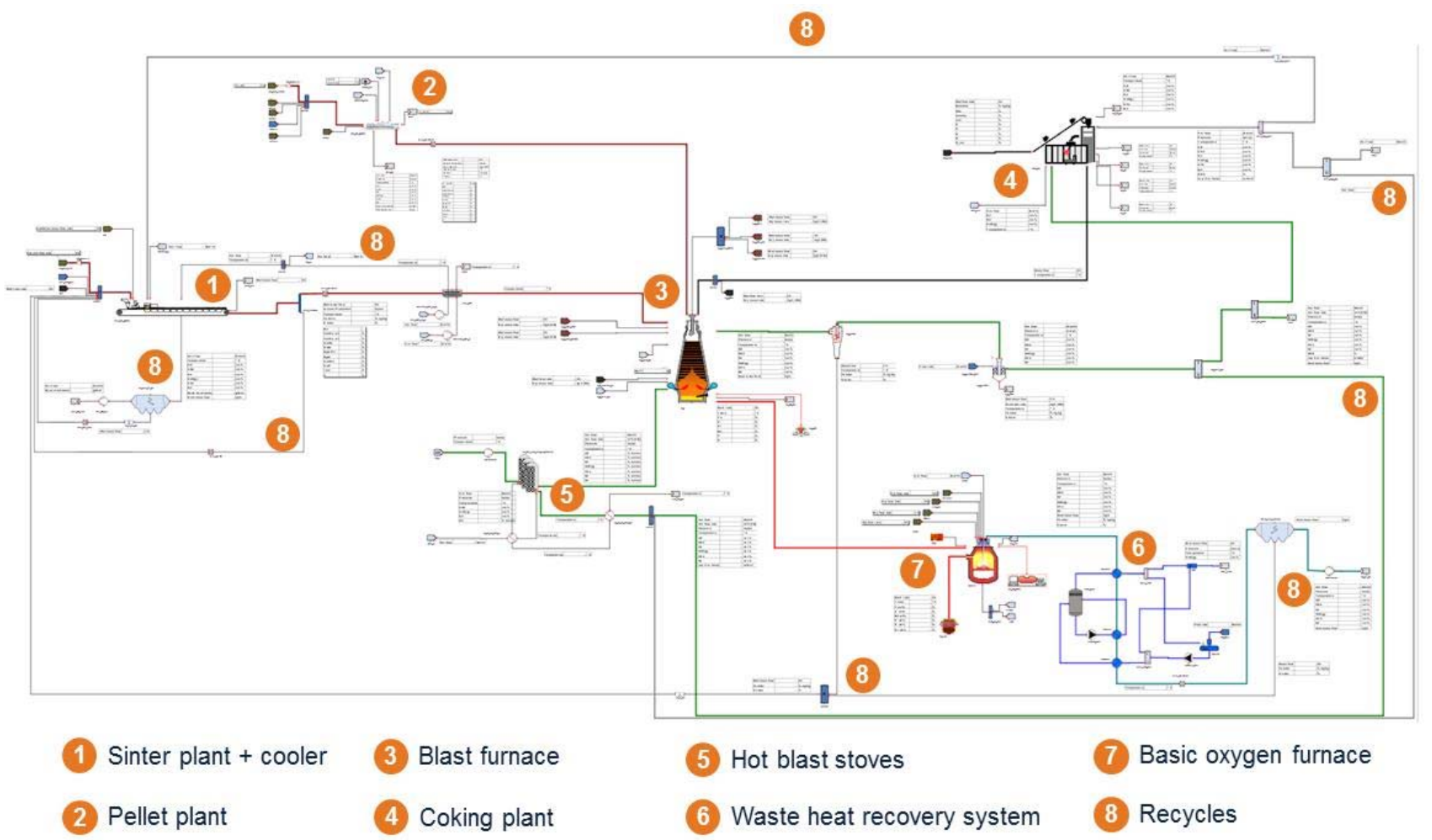

Figure no. 5: Example for a typical output flowsheet of the plant calculation model

\section{CONCLUSION}

The applications, where detailed analyses and improvements of the iron and steel making process are required, are increasing continuously in a market, in which the profitability of the operating plants is more and more endangered and the availability and utilization of high grade raw materials at reasonable cost rates is rather the exception. In the past the main effort has been focused on improving the downstream processes for iron and steel making and the potential for further developments are limited. In comparison to that the upstream processes and here the beneficiation in particular promise much more chances for improvements in order to make the downstream processes more viable by supplying higher grade raw materials at acceptable costs. 
Primetals Technologies accommodated to this situation by creating an environment to execute extensive test work from the run of mine iron ore to the liquid steel and is by that well prepared to provide beside its plants and technologies the optimum overall solutions for iron and steel making. To this prospects also contributes the Primetals plant calculation model, which enables to consider the whole steel making process from the run of mine ore downstream as one holistic unit without any inconsistencies and efficiency losses between the individual plants. 